\title{
Plasmon-driven reaction controlled by the number of graphene layers and localized surface plasmon distribution during optical excitation
}

\author{
Zhi-gao Dai ${ }^{1,2}$, Xiang-heng Xiao" ${ }^{1}$, Wei Wu ${ }^{2}$, Yu-peng Zhang ${ }^{1}$, Lei Liao ${ }^{1}$, Shi-shang Guo ${ }^{1}$, Jian-jian Ying ${ }^{1}$, \\ Chong-xin Shan ${ }^{3,4}$, Meng-tao Sun ${ }^{5}$ and Chang-zhong Jiang ${ }^{1}$
}

Graphene-plasmonic hybrid platforms have attracted an enormous amount of interest in surface-enhanced Raman scattering (SERS); however, the mechanism of employing graphene is still ambiguous, so clarification about the complex interaction among molecules, graphene, and plasmon processes is urgently needed. We report that the number of graphene layers controlled the plasmon-driven, surface-catalyzed reaction that converts para-aminothiophenol (PATP)-to- $p, p^{\prime}$-dimercaptoazobenzene (DMAB) on chemically inert, graphene-coated, silver bowtie nanoantenna arrays. The catalytic reaction was monitored by SERS, which revealed that the catalytic reaction occurred on the chemical inertness monolayer graphene $(1 \mathrm{G})$-coated silver nanostructures. The introduction of $1 \mathrm{G}$ enhances the plasmon-driven surface-catalyzed reaction of the conversion of PATP-to- $p, p^{\prime}$-DMAB. The chemical reaction is suppressed by bilayer graphene. In the process of the catalytic reaction, the electron transfer from the PATP molecule to 1G-coated silver nanostructures. Subsequently, the transferred electrons on the graphene recombine with the hot-hole produced by the localized surface plasmon resonance of silver nanostructures. Then, a couple of PATP molecules lost electrons are catalyzed into the $p, p^{\prime}$-DMAB molecule on the graphene surface. The experimental results were further supported by the finite-difference time-domain method and quantum chemical calculations.

Light: Science \& Applications (2015) 4, e342; doi:10.1038/lsa.2015.115; published online 23 October 2015

Keywords: graphene; graphene-mediated SERS; plasmon-driven reaction; SERS

\section{INTRODUCTION}

Since it was discovered in 2004, graphene, a single atomic layer of graphite, has attracted vast interest due to its unique properties ${ }^{1}$. Recently, the two vibrant and rich fields of investigation of graphene and plasmonics have strongly overlapped ${ }^{2}$. A combination of graphene with plasmonic nanostructures has substantially improved the photodetection capabilities of graphene $e^{3,4}$. The combination of graphene with conventional plasmonics based on noble metals could be beneficial for both fields of investigation: plasmonic nanostructures can enhance the properties of graphene (stronger Raman signature and more effective graphene-plasmonic photocells), and graphene can influence the optical response of plasmonic nanoarrays ${ }^{2,5,6}$. Because the interaction between light and graphene is determined by the local electromagnetic fields (induced on the graphene sheet), this interaction can be increased by placing metal nanostructures close to the graphene, such as well-defined dimensions gold nanodisks arrays on the top of graphene ${ }^{7}$, nanodisks arrays ${ }^{8}$, gold nanopyramids ${ }^{9}$, a photonic crystal nanocavity ${ }^{10}$, and a pair of gold nanodisks ${ }^{11}$ covered with graphene.
From a surface-enhanced Raman scattering (SERS) point of view, graphene provides the ideal prototype test material for investigation of SERS $^{12}$. In a graphene-mediated SERS (G-SERS) substrate ${ }^{13-18}$; the monolayer graphene $(1 \mathrm{G})$ provides an atomically flat surface for Raman enhancement. Because the graphene surface is chemically inert, signals from G-SERS substrates have great advantages over normal SERS by providing cleaner vibrational information free from various metal-molecule interactions (molecules cannot interact with the chemically active metal by graphene-mediated interactions) and being more stable against photo-induced damage ${ }^{13}$. Furthermore, the ultrasensitive graphene-plasmonic hybrid platform for label-free SERS detection is approaching single-molecule detection ${ }^{18}$.

However, previous studies ignored the fact that molecules can interact with (can even be photocatalyzed by) the chemically active metal through the high electron mobility of graphene during charge transfer in SERS measurements. Furthermore, the mechanism of SERS employing graphene has not been fully explored. Recently, increasing attention has turned to graphene-molecule ${ }^{12,19,20}$, moleculemetal $^{21,22}$, and metal-grapheme ${ }^{7-11}$ interactions; however, a great

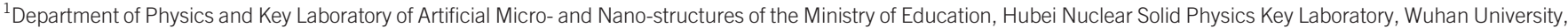

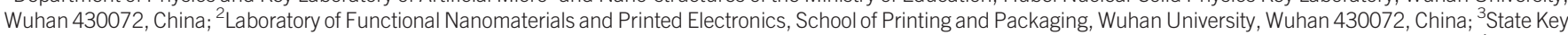

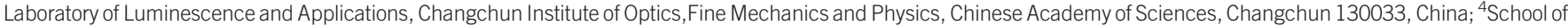

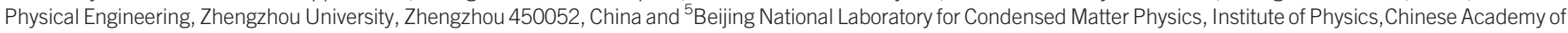
Sciences, Beijing 100190, China

Correspondence: XH Xiao, Email: xxh@whu.edu.cn; CX Shan, Email: shancx@ciomp.ac.cn; MT Sun, Email: mtsun@iphy.ac.cn

Received 30 January 2015; revised 2 June 2015; accepted 10 June 2015; accepted article preview online 20 June 2015 
challenge remains and clarification is needed. Currently, little is known about the complex interaction among molecules, graphene, and metal processes. This is the motivation for this work.

In this paper, we start by devising graphene-coated on Ag bowtie nanoantenna arrays (ABNA), as shown in Figure 1, where the paraaminothiophenol (PATP) on the substrate is an increasingly important probe molecule in SERS because of the intense SERS signal ${ }^{23-26}$. PATP-to- $p, p^{\prime}$-dimercaptoazobenzene (DMAB) conversion is used to study charge transfer between the metal and molecules ${ }^{27-31}$, during which the catalytic reaction is monitored by SERS. Large-area, well-ordered, uniform-sized ABNA replaces previously disordered gold/silver nanoislands as the SERS substrate. Well-ordered ABNAs can insure the same large-area graphene-metal interaction. The PATP-to-DMAB conversion is achieved on the chemically inert and high electron mobility 1G-coated on ABNA. The $1 G$ further enhances the plasmon-driven, surface-catalyzed reaction of the conversion of PATP-to-DMAB. In contrast, the conversion probability is lower on bilayer grapheme (2G)-coated on ABNA. The chemical reaction can be controlled by the distribution of localized surface plasmon. This discovery represents an important advancement toward the understanding of the complexity of the interaction among molecules, graphene, and metal processes and is also important for both plasmon-driven photocatalysis and applications of graphene. Furthermore, the DMAB molecule is an important organic optical electronic and molecular wire material ${ }^{32}$. There are potentially new photoelectric characteristics and potential applications of DMAB-graphene-plasmonic composites in transparent conductors and photodetection.

\section{MATERIALS AND METHODS}

Figure 1a is a schematic view of the graphene-assisted, plasmon-driven reaction of the transformation of PATP-to-DMAB. Layer-controlled graphene by chemical vapor deposition (CVD) was transferred to the ABNA. Large-area, well-ordered, uniform-sized ABNAs are presented in Figure $1 \mathrm{~b}$ (above the yellow dashed line). The yellow dashed line shows the graphene border. Graphene-coated ABNA is shown in Figure $1 \mathrm{~b}$ (below the yellow dashed line). A typical Raman spectrum of graphene-coated ABNA is shown in Supplementary Fig. S1. Only the $\mathrm{G}$ and $2 \mathrm{D}$ bands are observed, which indicate that the graphene is still of high quality on ABNA. After a month, bare Ag nanoparticles show large morphological changes without the 1G, as show in Figure 1d. However, the chemically inert, $1 \mathrm{G}$-covered Ag nanoantennas did not show signs of changes; the surfaces of the particles kept their triangle shape, as shown in Figure 1e. In a previous study, by passivating the surface of silver nanostructures with $1 \mathrm{G}$, atmospheric sulfur-containing compounds (hydrogen sulfide $\left(\mathrm{H}_{2} \mathrm{~S}\right)$ and carbonyl sulfide) were unable to degrade the surface of the silver ${ }^{33}$. The ABNA is protected by the graphene monolayer. The $\mathrm{H}_{2} \mathrm{~S}$ molecule is smaller and more active than the PATP molecule. Therefore, ABNA cannot come into contact with the PATP molecules covered with the graphene without laser exposure.

\section{RESULTS AND DISCUSSION}

\section{PATP-to-DMAB conversion on chemically inert graphene} on ABNA

The time-dependent PATP-to-DMAB conversions were monitored in situ by SERS on the ABNA, 1G and 2G-coated ABNA, respectively. PATP was deposited on the surface of the samples by thermal evaporation. The SERS spectrum of PATP on ABNA is seen in Figure 2a (SERS spectrum was collected with an exposure time of $10 \mathrm{~s}$ and a laser power of $3 \mathrm{~mW}$ ). The characteristic Raman peaks of DMAB are at 1387 and $1432 \mathrm{~cm}^{-1}$, which correspond to the $\mathrm{ag}_{16}$ and $\mathrm{ag}_{17}$ vibrational modes of $\mathrm{N}=\mathrm{N}$ of $\mathrm{DMAB}^{28,29,34,35}$. Therefore, the ratio of $I_{\mathrm{nn}} / I_{\mathrm{cc}}$ was observed to monitor the catalytic reaction, where $I_{\mathrm{nn}}$ and $I_{\mathrm{cc}}$ are the Raman intensities of the $1432 \mathrm{~cm}^{-1}$ band (from the generated $D M A B$ ) and the $1589 \mathrm{~cm}^{-1}$ band (from the benzene ring of the generated DMAB and unreacted PATP), respectively. The PATP-toDMAB conversion is almost completed on ABNA with prolonged irradiation time of $11 \mathrm{~min}$, as shown in Figure 2a.

The characteristic Raman peaks of DMAB were also found on PATP on the 1G-coated ABNA after a shorter time of $1 \mathrm{~min}$ (11 min for ABNA without graphene), indicating that the PATP-to-DMAB conversion occurred on the chemical inertness and high electron mobility $1 G$ on the ABNA. For comparison, the time-dependent

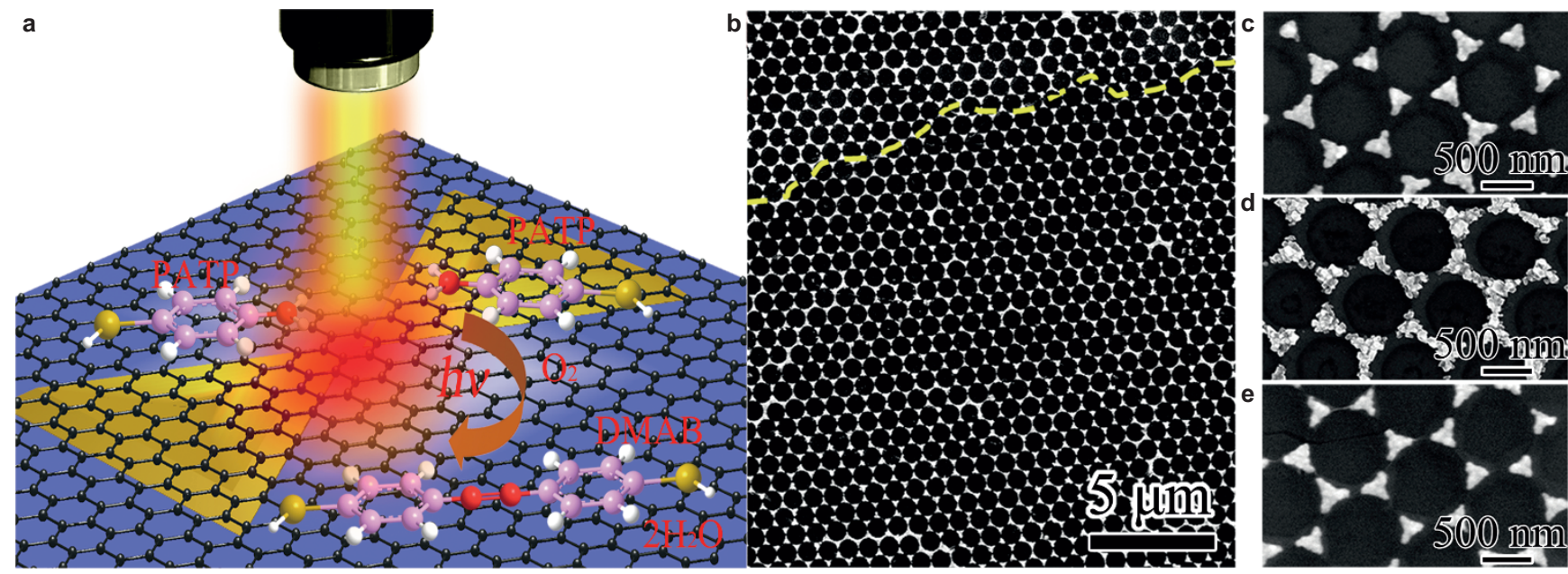

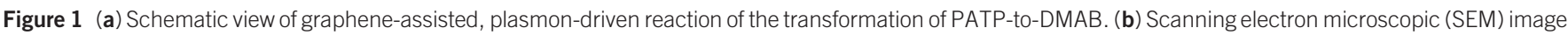

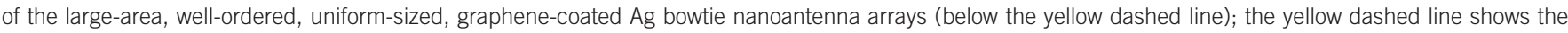

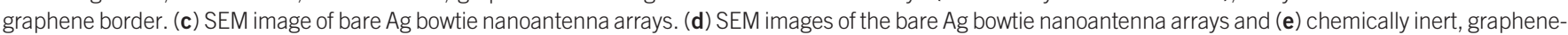
covered Ag nanoantenna arrays after a month. The Ag bowtie nanoantenna arrays are protected by the graphene monolayer. 

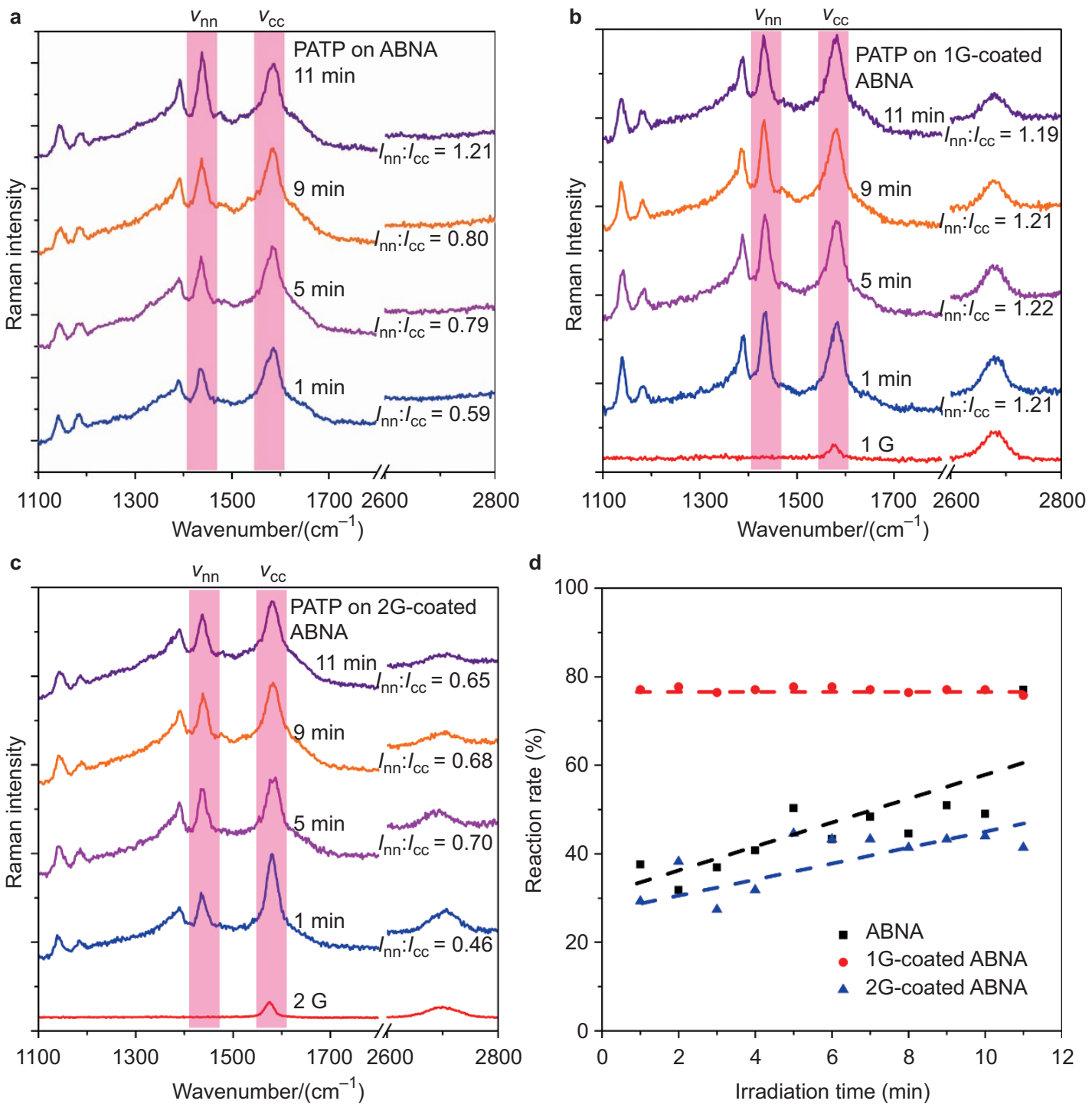

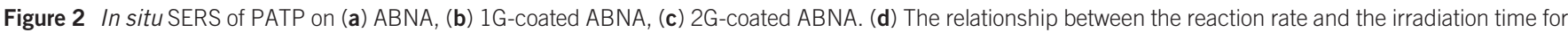
ABNA (black line), 1G-coated ABNA (red line), and 2G-coated ABNA (blue line).

normal Raman spectra of PATP on Si and graphene are also shown in Supplementary Fig. S2. The Raman spectrum of PATP on graphene shows that the vibrational modes at 1084 and $1589 \mathrm{~cm}^{-1}$ are $v_{\mathrm{cc}}+v_{\mathrm{cs}}$ and $v_{\mathrm{cc}}{ }^{29,36}$, respectively (the Raman spectra were collected with an exposure time of $60 \mathrm{~s}$ and a laser power of $10 \mathrm{~mW}$ ). Only the $\mathrm{G}$ (superposed with the $v_{\mathrm{cc}}$ of PATP) and 2D bands are observed, indicating that PATP deposition does not affect the quality of our synthesized graphene. A comparison between these two spectra of PATP on graphene and $1 \mathrm{G}$-coated ABNA shows that PATP cannot covert to DMAB on chemically inert graphene without metal, even though a higher laser power is employed. Furthermore, Zhang's group reported the Raman spectra of a self-assembled monolayer of PATP on a flat Au and Ag surface by graphene-mediated SERS tapes ${ }^{13}$. Although PATP was anchored on the flat gold surface and had been isolated from the hot spot ( $\mathrm{Ag}$ and $\mathrm{Au}$ nanoparticle gap) by graphene that was one atom thick, the weak and ignored characteristic Raman peaks of DMAB still emerged at approximately 1390 and $1430 \mathrm{~cm}^{-1}$. Therefore, the plasmon-driven transformation of PATP-to-DMAB occurs on graphene-covered Ag nanoparticles.
The number of graphene layers controlled the plasmon-driven reaction on graphene-covered $\mathrm{ABNA}$

To investigate the mechanisms of the graphene plasmon-driven, surface-catalyzed reaction of the conversion of PATP-to-DMAB, the

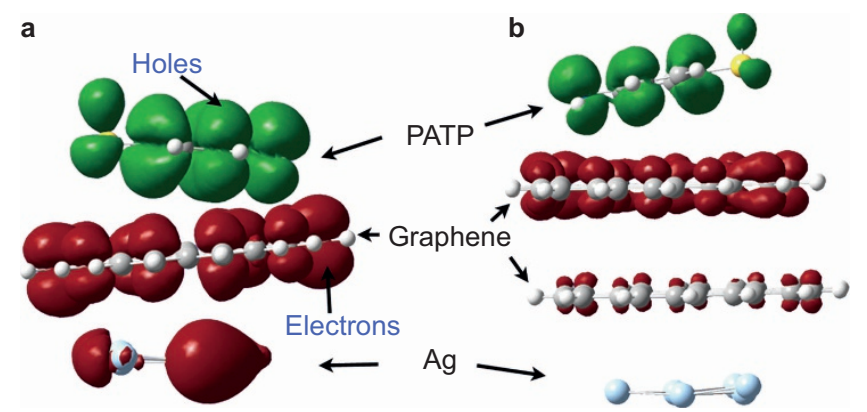

Figure 3 Charge difference densities for the electronic transitions of PATA/1G/ ABNA (a) and PATA/2G/ABNA (b), where green and red stands for holes and electrons, respectively. 

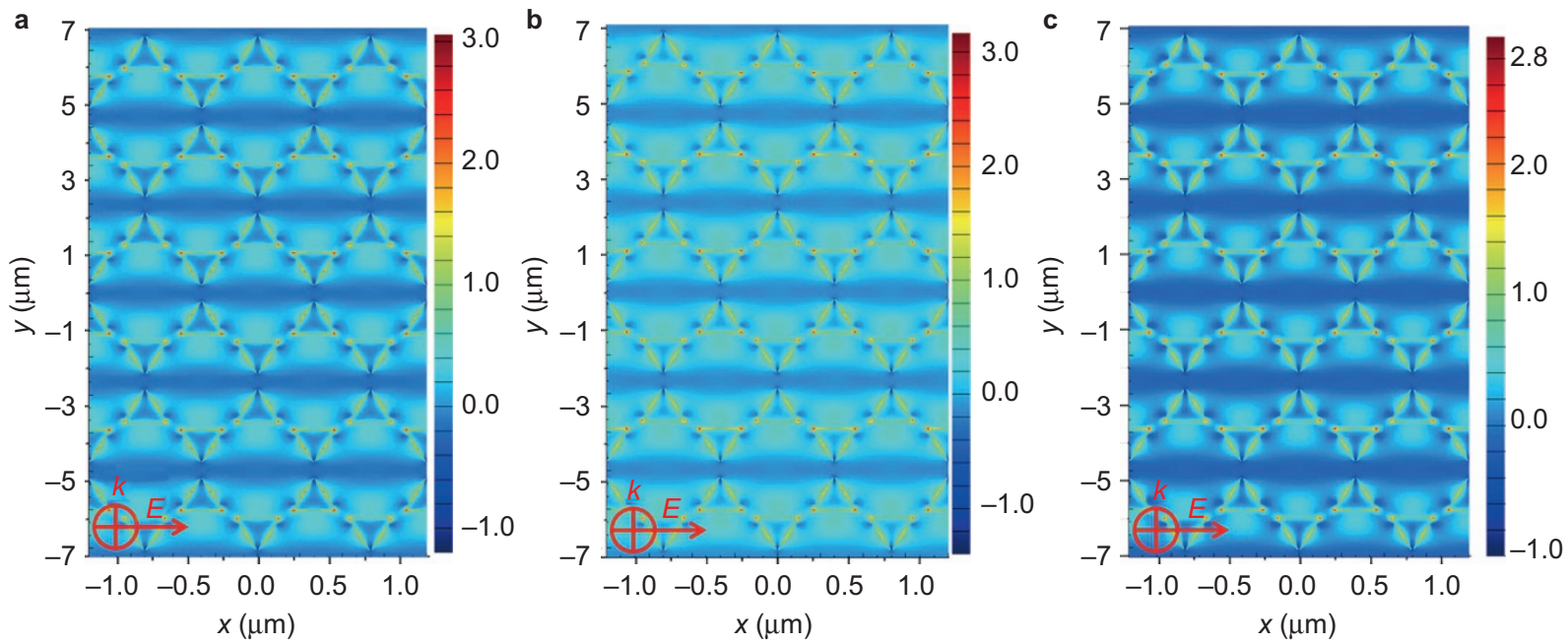

Figure 4 The FDTD calculated local electric field enhancement $\left(\log |\boldsymbol{E}|^{2}\right)$ of (a) ABNA, (b) 1G-coated ABNA, (c) 2G-coated ABNA at the XY plane at an incident wavelength of $488 \mathrm{~nm}$.

charge difference densities of PATP on graphene-coated Ag nanostructures were calculated. The orientation of the charge transfer for electronic transitions has been extensively studied by charge difference density $^{36-38}$. As a single sheet of $\mathrm{sp}^{2}$ carbon atoms, the delocalized $\pi$-bonds of graphene act as a natural 'magnet' for collecting aromatic molecules, where the aromatic molecules lie parallel to the graphene. Electrons can efficiently transfer from PATP to graphene and Ag clusters with excited states near $488 \mathrm{~nm}$ (see Figure 3a).

Furthermore, by comparing the $I_{\mathrm{nn}} / I_{\mathrm{cc}}$ of the two Raman spectra of PATP on ABNA and 1G-coated ABNA within 1 min, we can see that the reaction probability of the conversion of PATP-to-DMAB is larger on the $1 \mathrm{G}$-coated ABNA, where $I_{\mathrm{nn}}$ and $I_{\mathrm{cc}}$ are the Raman intensity of the $1432 \mathrm{~cm}^{-1}$ band (from the generated DMAB) and the Raman intensity of the $1589 \mathrm{~cm}^{-1}$ band (from the benzene ring of the generated DMAB, unreacted PATP and graphene(very weak)), respectively. For a more quantitative comparison, the spatial distributions of the electromagnetic field intensity for ABNA and 1G-coated ABNA (as measured by scanning electron microscope (SEM)) at the $X Y$ plane are calculated with the finite-difference time-domain (FDTD) method, using periodic boundary conditions (see Figure $4 \mathrm{a}$ and $4 \mathrm{~b}$ ). The 1G-coated ABNA has similar resonance positions to those of ABNA, but the electromagnetic enhancement $|\boldsymbol{E}|^{2}$ on the tip of the nanoparticle at an incident wavelength of $488 \mathrm{~nm}$ for $1 \mathrm{G}$-coated ABNA is stronger than that for $\operatorname{ABNA}\left(1.5 \times 10^{3}\right.$ versus $\left.1.1 \times 10^{3}\right)$, where $|\boldsymbol{E}|=\left|\boldsymbol{E}_{\text {local }} / \boldsymbol{E}_{\text {in }}\right|$ and $\boldsymbol{E}_{\text {local }}$ and $E_{\text {in }}$ are the local and incident electric fields, respectively. Similarly, Xu et al. reported the maximum $|\boldsymbol{E}|^{2}$ value in the graphene-containing gold hemisphere dimer is twice as large as that of the pristine gold hemisphere dimer ${ }^{13}$. In previous studies, a dielectric $\mathrm{SiO}_{2}$ layer introduced additional enhancement through the multi-scattering process ${ }^{39}$. A similar case may also exist in graphene-containing metal nanostructures. Thus, stronger resonance plasmon can be obtained, and more energetic, 'hot' electron-hole pairs ${ }^{40,41}$ are produced on $1 \mathrm{G}$-coated ABNA. Conversion of PATP-to-DMAB takes place with higher probability on 1G-coated ABNA. Therefore, $1 \mathrm{G}$ can enhance the plasmon-driven surface-catalyzed reaction of the conversion of PATP-to-DMAB.

For the 2G-coated ABNA, the ratio of $I_{\mathrm{nn}}$ to $I_{\mathrm{cc}}$ is still low after the longest irradiation time of $11 \mathrm{~min}$, as shown in Figure 2c. The relationships between the reaction rate and the irradiation time for ABNA (black line), 1G-coated ABNA (red line), and 2G-coated ABNA (blue line) are shown in Figure $2 \mathrm{~d}$. This result indicated that the reaction probability of the conversion of PATP-to-DMAB is small on 2G-coated ABNA. The characteristic Raman peaks of DMAB are found on the $1 \mathrm{G}$-coated ABNA with a more efficient reaction in $1 \mathrm{~min}$. The spatial distributions of the electromagnetic field intensity for 2G-coated ABNA at the $X Y$ plane were also calculated by the FDTD method using periodic boundary conditions (see Figure 4c). The electromagnetic enhancement $|\boldsymbol{E}|^{2}$ on the tip of the nanoparticle at an incident wavelength of $488 \mathrm{~nm}$ for $2 \mathrm{G}$ coated ABNA is slightly weaker than that for ABNA $\left(0.9 \times 10^{3}\right.$ versus $\left.1.1 \times 10^{3}\right)$. Similarly, the increase of the $\mathrm{SiO}_{2}$ layer thickness compromises the SERS activity ${ }^{39}$. Part of the reason for the smallest reaction probability is the least hot electron generation on the 2G-coated ABNA.

More importantly, the charge difference densities of PATP on the bilayer graphene on the Ag clusters were calculated. For the two-layer case, electrons can efficiently transfer only to the top of the bilayer graphene where the molecule is adsorbed, and the electrons cannot efficiently transfer to the Ag clusters and the bottom of the bilayer graphene, which is directly adsorbed on the Ag clusters (see Figure 3b). Thus, the bilayer graphene effectively decreases the probability of the PATP-to-DMAB conversion.
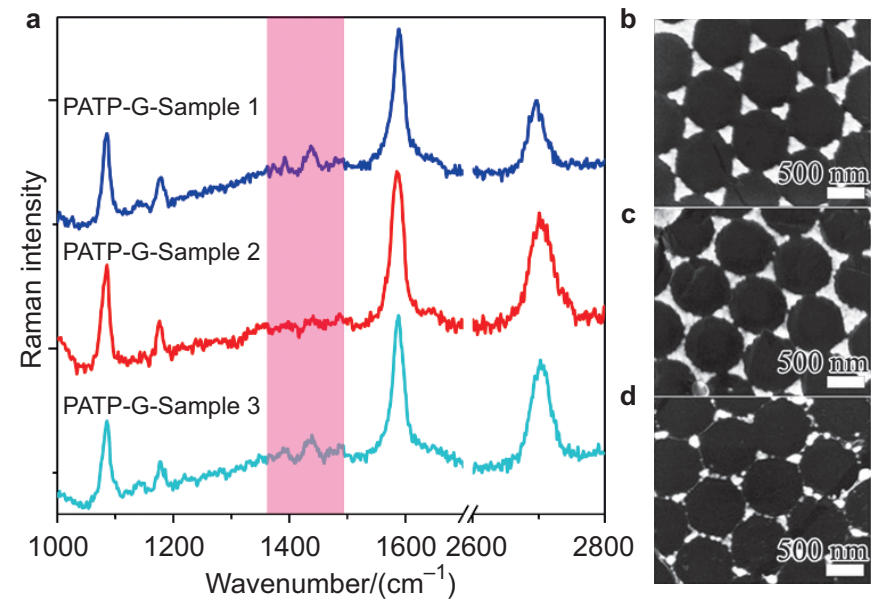

Figure 5 (a) The SERS spectra of PATP on graphene-covered sample 1, sample 2, and sample 3. And sample 1 (b), sample 2 (c), and sample 3 (d) corresponding SEM. 

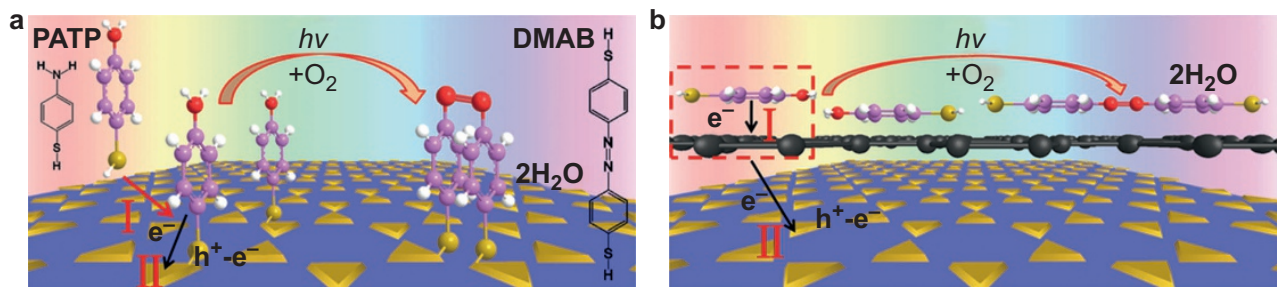

Figure 6 (a) Schematic view of the structure of PATP and the formation process of DMAB by the conventional plasmon-driven reaction. (b) Schematic view of the formation process of DMAB by the graphene-assisted, plasmon-driven reaction.

Process of the graphene enhanced plasmon-driven, surface-catalyzed reaction

To further investigate the PATP-graphene composite interaction with ABNA, three types of changed ABNA were fabricated. $\mathrm{SiO}_{2}$ films approximately $2 \mathrm{~nm}$ and $5 \mathrm{~nm}$ in thickness were deposited on asprepared ABNA prepared by radio frequency magnetron sputtering to obtain samples 1 and 2. For sample 3, the previous thickness of silver film of approximately $60 \mathrm{~nm}$ was decreased to $20 \mathrm{~nm}$. The graphene was transferred onto the three samples before PATP deposition. The SEM of graphene-covered sample 1, sample 2, and sample 3 are shown in Figure 5. The SERS spectra of PATP on the three graphene-coated samples are shown in Figure 5a. The characteristic Raman peaks of

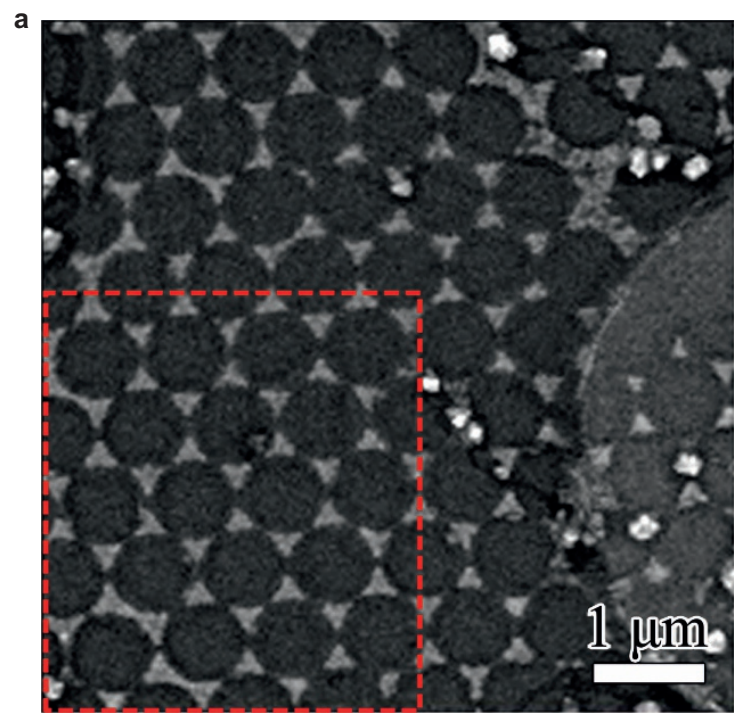

b
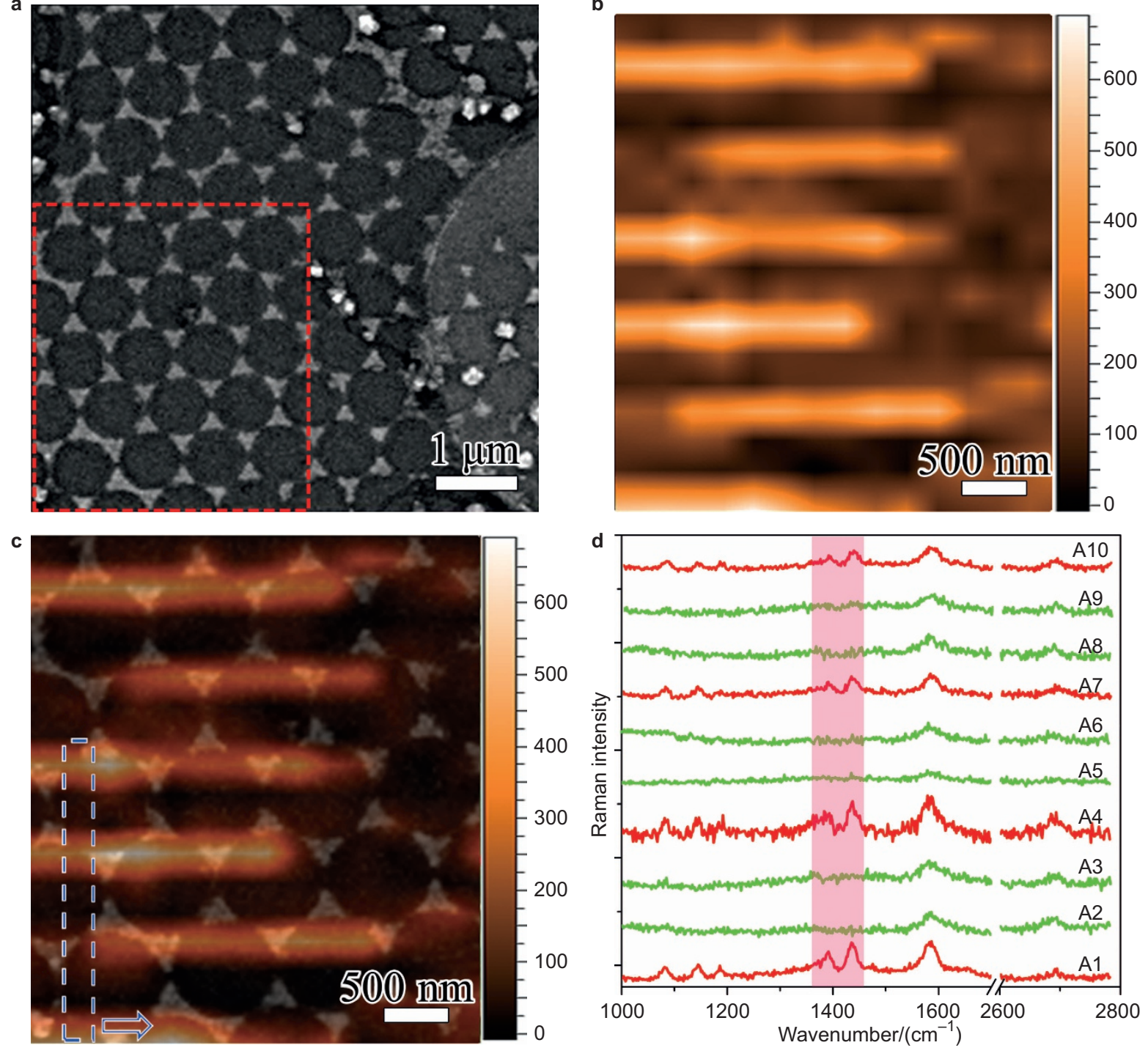

Figure 7 (a) The SEM of the randomly selected Raman mapping area of $3.5 \times 3.75 \mu \mathrm{m}^{2}$ marked by red dotted lines. The semi-circle is a marker of the UV lithographydefined coordinate system. (b) SERS intensity maps at $1432 \mathrm{~cm}^{-1}\left(v_{\mathrm{nn}}\right)$ corresponding to the area shown in (a). (c) overlay image of (a) and (b). (d) A list of randomly selected Raman spectra; their corresponding SEM and Raman mappings are marked by blue dotted lines, as shown in (c). 
DMAB $\left(1387 \mathrm{~cm}^{-1}\right.$ and $\left.1432 \mathrm{~cm}^{-1}\right)$ rise to different extents for the three samples. For samples 1 and 3 , the conversion of PATP-to-DMAB occurred within certain limits. However, for sample 2, the conversion of PATP-to-DMAB minimally occurred. Therefore, the thicker $\mathrm{SiO}_{2}$ film can prevent electrons from the PATP-graphene composite from recombining with strong hot-holes arising from the localized surface plasmon resonance (LSPR) of the ABNA as in samples 1 and 2 . The lesser produced hot-holes limit PATP-to-DMAB conversion in sample 3.

In previous studies ${ }^{28.29,36}$, the conventional plasmon-driven reaction of the conversion of PATP-to-DMAB consisted of two processes, as confirmed by ultrahigh-vacuum, tip-enhanced Raman spectroscopy ${ }^{28}$ shown in Figure 6a. The first process is the chemical adsorption of the PATP molecules onto the silver nanoparticles through a thiol group. The second process is that a couple of PATP molecules lose electrons to hot-holes arising from $\mathrm{LSPR}^{42,43}$; then the couple of PATP molecules convert to a DMAB molecule. For the graphenecontrolled, plasmon-driven, surface-catalyzed reaction, the following mechanism is demonstrated: electron transfer first from the PATP molecule to graphene; next, the electrons recombine with hot-holes arising from LSPR on the ABNA surface ${ }^{42,43}$; the $1 \mathrm{G}$ introduces a strong dipole allowed transition between PATP and graphene, which is enhanced by the EM fields; then, a couple of the PATP molecules lose electrons to become DMAB molecules on the graphene surface, as shown in Figure 6b. For the bilayer graphene, the electronic transition is weak and hot electron transfer from Ag is difficult.

\section{The distribution of the ABNA effected plasmon-driven reaction}

To demonstrate the large-area, ordered, graphene-controlled, plasmon-driven, surface-catalyzed reaction, which is dependent on the localized surface plasmon distribution, point-by-point Raman mapping was recorded on a randomly selected $3.5 \mu \mathrm{m} \times 3.75 \mu \mathrm{m}$ area with a step size of $250 \mathrm{~nm}$ on the 1G-coated ABNA. The SEM of the selected area is shown in Figure 7a marked by red dotted lines. By marking the silicon substrates with a UV lithography-defined coordinate system, we are able to observe, in the optical microscopy of the Raman system, the same field of view identified in the SEM.

First, the selected area is $1 \mathrm{G}$-coated ABNA confirmed by the $2 \mathrm{D}$ to $\mathrm{G}$ peak ratio Raman mapping. After PATP deposition, the Raman intensity mapping at $1432 \mathrm{~cm}^{-1}$ (the characteristic Raman peak of $D M A B)$ is shown in Figure $7 b$. As illustrated in Figure $7 b$, the graphene-assisted, plasmon-driven, surface-catalyzed reaction occurred periodically on well-ordered ABNA covered by large-area 1G. The regions of chemical reaction distribute at the croci strips in the same way as the hot spots are distributed in Figure 4. The overlay (Figure 7a and $7 \mathrm{~b}$ ) of the Raman mapping with the corresponding SEM shows more clearly that PATP-to-DMAB conversion mostly occurs on the $1 G$-covered bowtie nanoantenna tip. A list of randomly selected Raman spectra, A0-A10, is shown in Figure 7d. The corresponding SEM and Raman maps of the spectra A0-A10 are marked by blue dotted lines, as shown in Figure 7c. The emergence of the characteristic Raman peaks of DMAB at 1387 and $1432 \mathrm{~cm}^{-1}$ change periodically with the $1 \mathrm{G}$-coated ABNA orderly distribution. The chemical reaction could be controlled by localized surface plasmon distribution.

\section{CONCLUSIONS}

We demonstrate that PATP can be converted to DMAB on chemically inert, graphene-coated, ABNA during optical excitation, which was monitored by in situ SERS spectra. The PATP-to-DMAB conversion occurred on monolayer and bilayer graphene-covered ABNA to different degrees. 1G can further enhance the plasmon-driven, surface-catalyzed reaction. Bilayer graphene decreased the probability of the PATP-to-DMAB conversion. The chemical reaction was controlled by the localized surface plasmon distribution, as confirmed by the Raman mapping. To reveal the mechanisms, the charge difference density shows that electrons efficiently transferred from the PATP molecule to the 1G-coated Ag nanostructures. Subsequently, electrons recombined with hot-holes arising from LSPR (LSPR is excited by incident light). Some of the PATP molecules lose electrons to become DMAB molecules on the graphene surface. The $1 \mathrm{G}$ enhanced and the bilayer graphene suppressed the plasmon-driven, surface-catalyzed reaction, which represents an important advancement toward the understanding of the complex interaction among molecules, graphene, and metal processes and is also important for both plasmon-driven photocatalysis and applications of graphene.

\section{ACKNOWLEDGEMENT}

This work was partially supported by the NSFC (51171132, U1260102, 51371131, 11375134, 11374353, and 91436102), NCET (12-0418), Hubei Provincial Natural Science Foundation (2011CDB270, 2012FFA042), Jiangsu Provincial Natural Science Foundation (BK20141217), Wuhan Planning Project of Science and Technology (2014010101010019), the Wuhan University Experiment Technology Project Funding, the Fundamental Research Funds for the Central Universities (2042015kf1012, 2042015gf0016) and Liaoning Key Laboratory of Semiconductor Light Emitting and Photocatalytic Materials.

1 Novoselov K, Geim A, Morozov S, Jiang D, Zhang Y et al. Electric field effect in atomically thin carbon films. Science 2004; 306: 666-669.

2 Grigorenko AN, Polini M, Novoselov KS. Graphene plasmonics. Nat Photonics 2012; 6: 749-758.

3 Liu Y, Cheng R, Liao L, Zhou H, Bai J et al. Plasmon resonance enhanced multicolour photodetection by graphene. Nat Commun 2011; 2: 579-585.

4 Echtermeyer T, Britnell L, Jasnos P, Lombardo A, Gorbachev R et al. Strong plasmonic enhancement of photovoltage in graphene. Nat Commun $2011 ; 2$ : 458-462.

5 Barnes WL, Dereux A, Ebbesen TW. Surface plasmon subwavelength optics. Nature 2003; 424: 824-830.

6 Maier SA. Plasmonics: Fundamentals and Applications. New York: Springer; 2007.

7 Schedin F, Lidorikis E, Lombardo A, Kravets VG, Geim AK et al. Surface-enhanced Raman spectroscopy of graphene. ACS Nano 2010; 4: 5617-5626.

8 Kravets VG, Schedin F, Jalil R, Britnell L, Novoselov KS et al. Surface hydrogenation and optics of a graphene sheet transferred onto a plasmonic nanoarray. J Phys Chem C. 2012; 116: 3882-3887.

9 Wang P, Zhang W, Liang O, Pantoja M, Katzer J et al. Giant optical response from graphene-plasmonic system. ACS Nano. 2012; 6: 6244-6249.

10 Gan X, Mak KF, Gao Y, You Y, Hatami F et al. Strong enhancement of light-matter interaction in graphene coupled to a photonic crystal nanocavity. Nano Lett2012; 12: 5626-5631.

11 Heeg S, Fernandez-Garcia R, Oikonomou A, Schedin F, Narula R et al. Polarized plasmonic enhancement by Au nanostructures probed through Raman scattering of suspended graphene. Nano Lett 2013; 13: 301-308.

12 Ling $\mathrm{X}, \mathrm{Xie} \mathrm{L}$, Fang $\mathrm{Y}, \mathrm{Xu} \mathrm{H}$, Zhang $\mathrm{H}$ et al. Can graphene be used as a substrate for Raman enhancement? Nano Lett 2010; 10: 553-561.

13 Xu W, Ling X, Xiao J, Dresselhaus MS, Kong J et al. Surface enhanced Raman spectroscopy on a flat graphene surface. Proc Natl Acad Sci USA 2012; 109: 9281-9286.

14 Hao Q, Wang B, Bossard JA, Kiraly B, Zeng $Y$ et al. Surface-enhanced Raman scattering study on graphene-coated metallic nanostructure substrates. J Phys Chem C 2012; 116: 7249-7254.

15 Xu W, Xiao J, Chen Y, Chen Y, Ling X et al. Graphene-veiled gold substrate for surfaceenhanced Raman spectroscopy. Adv Mater 2013; 25: 928-933.

$16 \mathrm{Xu} \mathrm{W,} \mathrm{Mao} \mathrm{N,} \mathrm{Zhang} \mathrm{J.} \mathrm{Graphene:} \mathrm{a} \mathrm{platform} \mathrm{for} \mathrm{surface-enhanced} \mathrm{Raman}$ spectroscopy. Small 2013; 9: 1206-1224.

17 Zhu X, Shi L, Schmidt MS, Boisen A, Hansen 0 et al. Enhanced light-matter interactions in graphene-covered gold nanovoid arrays. Nano Lett 2013; 13: 46904696.

18 Wang $\mathrm{P}$, Liang $\mathrm{O}$, Zhang W, Schroeder T, Xie YH. Ultra-sensitive graphene-plasmonic hybrid platform for label-free detection. Adv Mater 2013; 25: 4918-4924.

$19 \mathrm{Xu} \mathrm{H}$, Xie L, Zhang H, Zhang J. Effect of graphene Fermi level on the Raman scattering intensity of molecules on graphene. ACS Nano 2011; 5: 5338-5344. 
20 Ling X, Moura LG, Pimenta MA, Zhang J. Charge-transfer mechanism in grapheneenhanced Raman scattering. J Phys Chem C 2012; 116: 25112-25118.

21 Petek H, Weida MJ, Nagano H, Ogawa S. Real-time observation of adsorbate atom motion above a metal surface. Science 2000; 288: 1402-1404.

22 Kale MJ, Avanesian T, Xin H, Yan J, Christopher P. Controlling catalytic selectivity on metal nanoparticles by direct photoexcitation of adsorbate-metal bonds. Nano Lett 2014; 14: 5405-5412.

23 Sun $\mathrm{M}, \mathrm{Xu} \mathrm{H}$. A novel application of plasmonics: plasmon-driven surface-catalyzed reactions. Small 2012; 8: 2777-2786.

24 Ward DR, Halas NJ, Ciszek JW, Tour JM, Wu Y et al. Simultaneous measurements of electronic conduction and Raman response in molecular junctions. Nano Lett 2008; 8: 919-924.

25 Zhou Q, Li X, Fan Q, Zhang X, Zheng J. Charge transfer between metal nanoparticles interconnected with a functionalized molecule probed by surface-enhanced Raman spectroscopy. Angew Chem Int Ed Engl 2006; 45: 3970-3973.

26 Ye J, Hutchison JA, Uji-i H, Hofkens J, Lagae L et al. Excitation wavelength dependent surface enhanced Raman scattering of 4-aminothiophenol on gold nanorings. Nanoscale 2012; 4: 1606-1611.

27 van Schrojenstein Lantman EM, Deckert-Gaudig T, Mank AJG, Deckert V, Weckhuysen BM. Catalytic processes monitored at the nanoscale with tip-enhanced Raman spectroscopy. Nature Nanotech 2012; 7: 583-586.

28 Huang YZ, Fang YR, Zhang ZL, Zhu L, Sun MT. Nanowire-supported plasmonic waveguide for remote excitation of surface-enhanced Raman scattering. Light: Sci App/2014; 3: e199; doi:10.1038/lsa.2014.80.

29 Sun MT, Zhang ZL, Wang PJ, Li Q, Ma FC et al. Remotely excited Raman optical activity using chiral plasmon propagation in Ag nanowires. Light: Sci App/ 2013; 2: e112; doi:10.1038/lsa.2013.68.

30 Xie W, Walkenfort B, Schlücker S. Label-free SERS monitoring of chemical reactions catalyzed by small gold nanoparticles using 3D plasmonic superstructures. J Am Chem Soc 2013; 135: 1657-1660.

31 Huang YF, Wu DY, Zhu HP, Zhao LB, Liu GK et al. Surface-enhanced Raman spectroscopic study of p-aminothiophenol. Phys Chem Chem Phys 2012; 14: 8485-8497.

32 Zhang $\mathrm{C}$, Du MH, Cheng HP, Zhang XG, Roitberg A et al. Coherent electron transport through an azobenzene molecule: a light-driven molecular switch. Phys Rev Lett 2004; 92: 158301-158304.
33 Reed JC, Zhu H, Zhu AY, Li C, Cubukcu E. Graphene-enabled silver nanoantenna sensors. Nano Lett 2012; 12: 4090-4094.

34 Huang YF, Zhu M, Zhao LB, Feng JM, Wu DY et al. Activation of oxygen on gold and silver nanoparticles assisted by surface plasmon resonances. Angew Chem Int Ed Engl 2014; 53: 2353-2357.

35 Dai Z, Xiao X, Zhang Y, Ren F, Wu W et al. In situ Raman scattering study on a controllable plasmon-driven surface catalysis reaction on $\mathrm{Ag}$ nanoparticle arrays. Nanotechnology 2012; 23: 335701-335705.

36 Fang YR, Li YZ, Xu HX, Sun MT. Ascertaining p,p'-dimercaptoazobenzene produced from $p$-aminothiophenol by selective catalytic coupling reaction on silver nanoparticles. Langmuir 2010; 26: 7737-7746.

37 Yan J, Henriksen EA, Kim P, Pinczuk A. Observation of anomalous phonon softening in bilayer graphene. Phys Rev Lett 2008; 101: 136804-136807.

38 Ataca C, Aktürk E, Ciraci S. Hydrogen storage of calcium atoms adsorbed on graphene: first-principles plane wave calculations. Phys Rev B 2009; 79: 041406-041410.

$39 \mathrm{Xu} \mathrm{H}$. Theoretical study of coated spherical metallic nanoparticles for single-molecule surface-enhanced spectroscopy. Appl Phys Lett 2004; 85: 5980-5982.

40 Knight MW, Sobhani H, Nordlander P, Halas NJ. Photodetection with active optical antennas. Science 2011; 332: 702-704.

41 Mukherjee S, Libisch F, Large N, Neumann O, Brown LV et al. Hot electrons do the impossible: plasmon-induced dissociation of $\mathrm{H}_{2}$ on Au. Nano Lett 2013; 13: 240-247.

42 Christopher P, Xin H, Linic S. Visible-light-enhanced catalytic oxidation reactions on plasmonic silver nanostructures. Nature Chem 2011; 3: 467-472.

43 Linic S, Christopher P, Ingram DB. Plasmonic-metal nanostructures for efficient conversion of solar to chemical energy. Nature Mater 2011; 10: 911-921.

\footnotetext{
(c) (i) (2) This license allows readers to copy, distribute and transmit the Contributionas long as it attributed back to the author. Readers are permitted to alter, transform or build upon the Contribution as long as the resulting work is then distributed under this is a similar license. Readers are not permitted to use the Contribution for commercial purposes. Please read the full license for further details at - http://creativecommons.org/ licenses/by-nc-sa/4.0/
} 